PULSAR MODELS

\section{Agreement af RAS}

by our Cosmology Correspondent

IN only three years pulsars have been reduced from the centre of attention among astronomers to the mundane level of routine work. This rapid decline was clearly evident at the specialist meeting on pulsars held by the Royal Astronomical Society on February 12, which contrasted sharply with the first RAS meeting after the announcement of the discovery of these unusual radio sources, or, more recently, with the similar specialist meeting on X-ray astronomy held in November last year (see Nature, 228, $715 ;$ 1970). At these earlier meetings, there was a great deal of variety in the ideas and observations presented, with many questions and other contributions from the floor. But at the more recent gathering, only different versions of the same basic model were presented, and very few new observations. The magic word "pulsar" still had sufficient charisma to attract a large audience, but this remained on the whole passive, so that the individual speakers and the whole meeting ran almost exactly to the planned schedule-a very unusual occurrence, and one which is not necessarily a good sign.

Before discussion of the theory of pulsar mechanisms, Professor F. Graham Smith (Jodrell Bank) presented the by now familiar data obtained from the addition of many successive pulses from a pulsar, which seem to show a variation of pulse arrival time over a "window" which remains constant and very well defined. Together with the polarization evidence, this suggests strongly that the regions producing pulsar radiation are confined to a local area (covering some $10^{\circ}$ in longitude) on the underlying neutron star; the size of this allowed area defines the observed window, and the small degree of variation in longitude within this region can explain the secular variation frequently observed over several successive pulses. Presumably, this variation is related to the degree of flexibility in the magnetic field which is now almost universally accepted as the beaming mechanism.

The first person to present the pulsar model was Professor L. Mestel (University of Manchester), who made the most of his opportunity to address a still receptive audience, outlining the oblique rotator model which stands unchallenged because of its ready explanation of the timing mechanism of pulsars. This model is based on the natural assumption that a rotating neutron star may have an associated magnetic field, and that the magnetic polar axis is unlikely to be the same as the star's spin axis. It is equally implausible that the magnetic axis would be at right angles to the spin axis, but consideration of these two extreme cases shows how the more probable intermediate cases may produce interactions leading to beamed radiation.

Streaming of charged particles outwards along the magnetic lines of force in this kind of model naturally leads to a situation where they are moving in a circle close to the speed of light, because although the magnetic field may lag behind the solid rotation of the underlying neutron star it must rotate almost as a solid body. Rotation at constant angular velocity then implies that sufficiently far from the star the field is rotating at the speed of light. The silent majority seemed happy to accept this idea as a now well established fact, especially when it was presented three times in rapid succession, with only relatively minor problems remaining to be solved. In one interpretation, the outward flow of plasma only reaches relativistic velocities (and produces beamed synchrotron radiation) where the magnetic field is travelling at twice the speed of light, and there was some discussion of the detail of the kind of magnetosphere which would agree with the observed radiation. To an outside observer the fire and excitement has definitely gone out of the pulsar hunt. Indeed, the experts are so blasé that they accepted the news that the Crab pulsar's so-called precursor pulse is completely absent at a frequency of $606 \mathrm{MHz}$ (see Nature Physical Science, 229, 192; 1971) without comment, even though this pulse is the strongest of the three seen in NP 0532 at half this frequency.

\title{
Identification of $\boldsymbol{X}$ and $\boldsymbol{Y}$-bearing Human Sperm
}

IT has been shown recently that some fluorescent dyes such as quinacrine mustard stain certain chromosome regions in a specific fashion. Of particular interest is the discovery that the distal end of the long arm of the human $Y$ chromosome has a very characteristic fluorescent band (L. Zech, Exp. Cell. Res., 58, 141; 1969) enabling its detection in mitotic and meiotic cells as well as in interphase nuclei. Last year, two groups of workers (P. L. Pearson and $\mathbf{M}$. Bobrow, J. Reprod. Fert., 22, 177; 1970; and P. Barlow and C. G. Vosa, Nature, 226, 961; 1970) reported that slightly less than 50 per cent of mature human sperm stained with quinacrine contain a fluorescent spot and these were tentatively identified as $Y$-bearing sperm.

In next Wednesday's Nature New Biology, Sumner, Robinson and Evans report their findings from a study of sperm from nine chromosomally normal individuals. Their results confirm that just under 50 per cent of mature sperm contain a fluorescent spot. In view of the fact that the human $X$ chromosome is much larger than the $Y$, they point out that $Y$-bearing sperm should contain significantly less DNA than $X$-bearing ones. Using fluorescent staining followed by optical density DNA measurements, they show that the sperm with the fluorescent spot do indeed contain significantly less DNA than those without fluorescence. This optical density difference is of the order of just below 3 per cent and fits in well with the estimates of approximately 4 per cent from published linear measurement of metaphase chromosomes as the difference in length and thus presumably in DNA content between the $X$ and the $Y$ chromosomes. The present DNA measurements thus confirm the value of the fluorescent technique in distinguishing $X$ and $Y$-bearing sperm.

In addition to the two major classes of sperm Sumner and his colleagues note that a small proportion (1.26 per cent) have two fluorescent spots and a DNA value intermediate between the $X$ and $Y$-bearing classes and conclude that these are $24, Y Y$ sperm which have arisen from $Y$ chromosome nondisjunction at the second division of meiosis. The complementary product of such a nondisjunction would be a sperm with a haploid autosome set and no sex chromosome. These two types of abnormal gamete would, if successful in fertilization, give rise to $47, X Y Y$ and $45, X$ zygotes. If all the 1 per cent or so of $24, Y Y$ and a similar number of sex chromosome deficient sperm were as successful at fertilization as normal sperm, a similar proportion of both $47, X Y Y$ and $45, X$ zygotes might be expected. Studies of live newborn populations indicate an incidence of 0.8 per thousand live births for $47, X Y Y$ and 0.2 per thousand live births for $45, X$ individuals.

The $X O$ sex chromosome complement is a very notable contribution to foetal loss (approximately 7 per cent of all spontaneous abortions), but a $47, X Y Y$ complement has not been described in an abortus. Because of the discrepancy between the proportions of presumptive $24, Y Y$ sperm and $47, X Y Y$ live births, and taking into account that there is no evidence of $47, X Y Y$ foetal loss, the authors conclude that $24, Y Y$ sperm have a reduced capacity for fertilization compared with normal sperm. This is of interest, as is the fact that there is a great excess of female 18 -trisomic $(47, X X, 18+)$ newborns and also a small excess of female abortuses with this trisomy suggesting that disomic-18 $Y$-bearing $(24, Y, 18+)$ sperm may be at a similar disadvantage.

The authors have also studied sperm from bull, rabbit and mouse and meiotic and mitotic cells from rabbit and mouse and found no strongly fluorescing chromosome in these species. 